\title{
Preferential recruitment of ataxin-3 independent of expanded polyglutamine: an immunohistochemical study on Marinesco bodies
}

\author{
H Fujigasaki, T Uchihara, J Takahashi, H Matsushita, A Nakamura, S Koyano, \\ K Iwabuchi, S Hirai, H Mizusawa
}

\begin{abstract}
Department of
Neuropathology, Tokyo Metropolitan Institute for Neuroscience, 2-6 Musashidai,

Fuchu-shi, Tokyo,

Japan

H Fujigasaki

T Uchihara

A Nakamura

S Koyano
\end{abstract}

Department of

Neuropathology, The

Jikei University, Japan

J Takahashi

Department of

Pathology, Toranomon

Hospital, Japan

H Matsushita

Department of

Neurology, Yokohama

City University, Japan

S Koyano

Department of

Psychiatry and

Neurology, Kanagawa

Rehabilitation Center,

Japan

K Iwabuchi

Department of

Neurology, Tokyo

Metropolitan

Neurological Hospital,

Japan

S Hirai

Department of

Neurology and

Neurological Science,

Tokyo Medical and

Dental University,

Graduate School of

Medicine, Japan

H Mizusawa

Correspondence to:

Dr T Uchihara

uchihara@tmin.ac.jp

Received 19 February 2001 and in revised form

2 May 2001

Accepted 16 May 2001

\begin{abstract}
In an immunohistochemical study of Marinesco bodies-a neuronal intranuclear inclusion often seen in neurons of the substantia nigra of patients with hepatic encephalopathy-it was shown that one of the polyglutamine proteins, ataxin-3, is preferentially recruited into this inclusion, whereas other polyglutamine proteins (ataxin-2 and TATA box-binding protein) are not. This suggests that recruitment of each of the polyglutamine proteins may be differently regulated. Because this nuclear inclusion is thought to be formed in response to cellular stress, as occurs in hepatic encephalopathy, even in the absence of an expanded CAGI polyglutamine repeat, recruitment of ataxin-3 and ubiquitin into Marinesco bodies may represent a cellular response to noxious external stimuli unrelated to expanded CAG/polyglutamine.

(F Neurol Neurosurg Psychiatry 2001;71:518-520)
\end{abstract}

Keywords: ubiquitin; hepatic encephalopathy; neuronal intranuclear inclusion

Ataxin-3 is the product of the gene responsible for Machado-Joseph disease (MJD)/ spinocerebellar ataxia (SCA) type 3, one of the dominantly inherited cerebellar ataxias. Abnormally expanded polyglutamine within ataxin-3 has been considered to be involved in the pathogenesis of this disease. Polyglutamine expansions are also responsible for other polyglutamine diseases: SCA1, 2, 6, and 7, Huntington's disease, dentatorubralpallidoluysian atrophy, and spinal and bulbar muscular atrophy. ${ }^{1}$ Recent studies have shown that the disease related proteins with abnormally expanded polyglutamine, except for the $\alpha-1 \mathrm{~A}$ calcium channel, form neuronal intranuclear inclusions (NIIs) in the affected brains. ${ }^{1-3}$ Neuronal intranuclear inclusions also contain ubiquitin, chaperon proteins, and proteins carrying non-expanded polyglutamine. ${ }^{145} \mathrm{We}$ have previously reported that Marinesco bodies, another example of ubiquitinated intranuclear inclusions found in pigmented neurons of the substantia nigra in normal people, also contain wild-type ataxin- $3 .^{6}$ This finding indicates that ataxin-3 is recruited into this intranuclear inclusion formed in the absence of expanded polyglutamine. The aim of this study is to extend this finding by looking for ataxin-3 and other polyglutamine proteins in Marinesco bodies that are abundant in brains from patients with hepatic encephalopathy.

\section{Materials and methods}

Five midbrains from patients with hepatic encephalopathy, mean age (SD) 59.4 (9.7), and five age matched controls without neurological disorders, mean age (SD) 58.8 (8.6), were examined. Age at death and pathological diagnosis are summarised in table 1 . Thick ( 6 $\mu \mathrm{m})$ sections of formalin-fixed midbrain were stained with haematoxylin and eosin, or immunostained with antibodies against ubiquitin (1:200, DF2, IgM monoclonal, a generous gift from Dr H Mori, Osaka City University), ataxin-3 (1:1000), ${ }^{6}$ ataxin-2 $(1: 200,15 \mathrm{~F} 6),{ }^{2}$ and TATA box binding protein (TBP) (1:1000, Santa-Cruz), ${ }^{4}$ under the conditions described previously. ${ }^{2}{ }^{4}$ The number of Marinesco bodies labelled by each staining was quantified by scanning a hemisubstantia nigra. The statistical significance of differences between the hepatic encephalopathy group and the control group were determined by the non-parametric Mann-Whitney $U$ test. The results obtained with the different staining methods were compared by analysis of variance (ANOVA). A double immunofluorescence study was performed with the antiubiquitin and antiataxin-3 antibodies, visualised by antimouse IgM coupled with $\mathrm{Cy}-5$ for the antiubiquitin antibody and antirabbit IgG coupled with FITC for the antiataxin-3 antibody. Confocal images were obtained with a Leica TCS-SP confocal laser microscope (Heidelberg, Germany).

\section{Results}

Many Marinesco bodies were seen on sections stained by haematoxylin and eosin (fig 1a), the antiubiquitin antibody (fig $1 \mathrm{~b}$ ), and the antiataxin-3 antibody (fig $1 \mathrm{c}$ ). Marinesco bodies detected by these three methods were quantified. The mean number of Marinesco bodies in the hepatic encephalopathy group 
Table 1 Summary of patients and numbers of Marinesco bodies

\begin{tabular}{|c|c|c|c|c|c|c|}
\hline & \multirow[b]{2}{*}{ Patients } & \multirow{2}{*}{$\begin{array}{l}\text { Age at } \\
\text { death }\end{array}$} & \multirow[b]{2}{*}{ Pathological diagnosis } & \multicolumn{3}{|c|}{ Numbers of Marinesco bodies } \\
\hline & & & & $H E$ & Ubiquitin & Ataxin-3 \\
\hline \multirow[t]{5}{*}{ Control } & 1 & 54 & Oesophageal cancer & 8 & 9 & 8 \\
\hline & 2 & 66 & Malignant lymphoma & 21 & 19 & 19 \\
\hline & 3 & 53 & Lung cancer & 29 & 34 & 37 \\
\hline & 4 & 51 & Cervical cancer & 28 & 34 & 30 \\
\hline & 5 & 70 & Myocardial infarction & 68 & 64 & 61 \\
\hline Mean & & 58.8 & & 30.8 & 32 & 31 \\
\hline Hepatic & 6 & 67 & Liver cirrhosis & 24 & 30 & 28 \\
\hline \multirow[t]{4}{*}{ Encephalopathy } & 7 & 66 & $\begin{array}{l}\text { Hepatocellular } \\
\text { carcinoma }\end{array}$ & 49 & 58 & 52 \\
\hline & 8 & 52 & Liver cirrhosis & 91 & 90 & 95 \\
\hline & 9 & 46 & $\begin{array}{l}\text { Hepatocellular } \\
\text { carcinoma }\end{array}$ & 30 & 35 & 23 \\
\hline & 10 & 66 & $\begin{array}{l}\text { Hepatocellular } \\
\text { carcinoma }\end{array}$ & 138 & 145 & 138 \\
\hline Mean & & 59.4 & & 66.4 & 71.6 & 67.2 \\
\hline
\end{tabular}

HE=haematoxylin and eosin.

(about 70 Marinesco bodies/case, table 1) was more than twice that of the control group (about 30 Marinesco bodies/case, table 1). The difference did not reach significance $(p>0.1$, Mann-Whitney $U$ test), however, because of the great variability within each group. No Marinesco bodies were labelled with the antiataxin-2 and anti-TBP antibodies despite the large number examined (data not shown). The number of Marinesco bodies visualised by haematoxylin and eosin, the antiataxin-3, or ubiquitin antibodies did not differ significantly either in the hepatic encephalopathy group or in the control group ( $p>0.9$ ANOVA). The localisation of ubiquitin differed from that of ataxin-3. Double immunofluorescence with the antiubiquitin and the antiataxin- 3 antibodies showed that ataxin-3 was localised in the central core of the Marinesco bodies surrounded by ubiquitin which appears as a ring on the sections (fig $1 \mathrm{~d}$, e, and $\mathrm{f}$ ).

\section{Discussion}

Marinesco bodies are the eosionophilic intranuclear inclusions seen in pigmented neurons of the substantia nigra. It has been reported
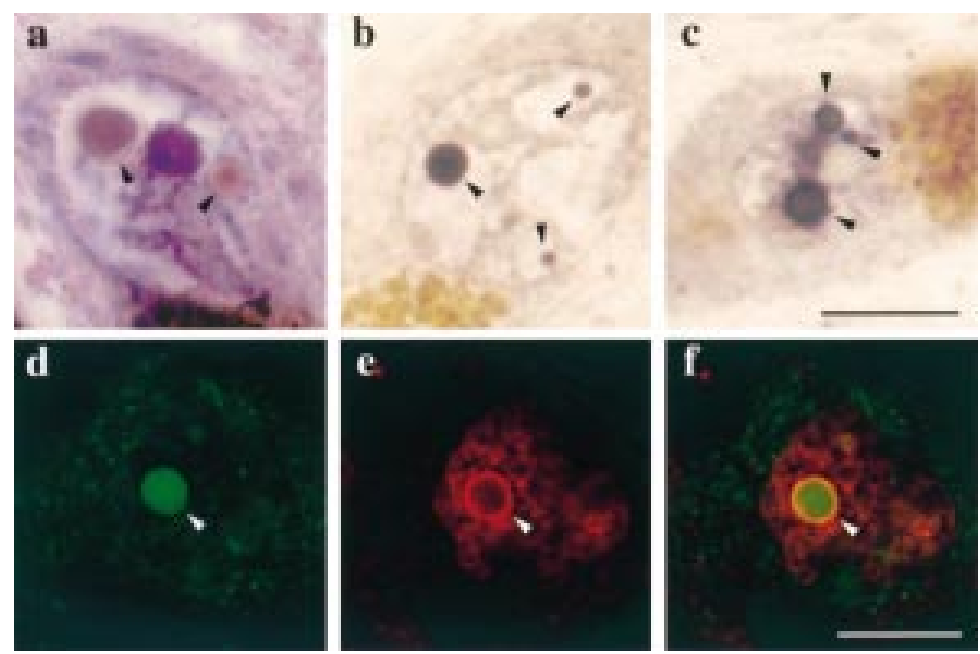

Figure 1 Immunohistochemical analysis and double immunofluorescence. (a) haematoxylin and eosin staining, (b) and (d) ataxin-3 immunoreactivity, (c) and (e) ubiquitin

immunoreactivity, $(f)$ merged image of $(d)$ and $(e)$. Antiataxin-3 antibody and antiubiquitin antibody recognised Marinesco bodies. Intense ubiquitin-like immunoreactivity was localised on the periphery of the Marinesco bodies (c). Ataxin-3 is localised in the central core of Marinesco bodies, whereas ubiquitin surrounds the core, appearing as a ring on the tissue sections $(d-f)$. These images of the same neuron $(d-f)$ were obtained by confocal microscopy. Arrowheads indicate Marinesco bodies (bars $=5 \mu \mathrm{m} ; \mathrm{a}-\mathrm{c}$ same magnification) that these bodies increase in number during aging $^{89}$ and in diseases such as hepatic encephalopathy. ${ }^{7}$ Although the pathological relevance of these inclusions has yet to be shown, their accumulation during aging or under pathological conditions such as hepatic encephalopathy suggests that formation of Marinesco bodies may be a cellular response to external stimuli. This hypothesis is supported by the fact that Marinesco bodies contain ubiquitin, known to be involved not only in stress responses ${ }^{10}$ but also in various neurodegenerative disorders. ${ }^{11}$ We previously showed that ataxin-3 is recruited into Marinesco bodies. ${ }^{6}$ The present study shows that almost all Marinesco bodies seen either in hepatic encephalopathy or in control groups contain ataxin-3 in common, indicating that it is one of the main components of this inclusion. Furthermore, neither ataxin-2 nor TBP were found in Marinesco bodies either in the hepatic encephalopathy group or in the control group, further indicating that Marinesco bodies are identical regardless of their pathological origin. Because proteins with non-expanded polyglutamine can be recruited into nuclear inclusion formed in the presence of another protein with pathologically expanded polyglutamine, it has been postulated that an interaction between expanded polyglutamine and nonexpanded polyglutamine may be a prerequisite for their nuclear translocation and the formation of NIIs. ${ }^{45}$ However, the consistent recruitment of wild-type ataxin-3 into Marinesco bodies indicates that another protein specific mechanism distinct from non-specific polyglutamine-polyglutamine interactions might mediate recruitment of wild type ataxin-3 into ubiquitinated intranuclear inclusions, as demonstrated previously. ${ }^{4}$ The absence of ataxin-2 and TBP in Marinesco bodies also supports the hypothesis that wild-type ataxin-3 may be recruited into ubiquitinated intranuclear inclusions by a protein specific mechanism, independent of a polyglutaminepolyglutamine interaction. Although the physiological and pathological functions of ataxin-3 remain to be elucidated, its preferential recruitment and colocalisation with ubiquitin in Marinesco bodies suggests that ataxin-3, like ubiquitin, is involved in cellular reactions to stress, as in hepatic encephalopathy or aging. The double immunofluorescence study clearly shows that ubiquitin almost always accumulated on the periphery of Marinesco bodies, whereas ataxin-3 was concentrated in the centre. This characteristic morphological feature is shared by NIIs of MJD/SCA3 brains as we reported elsewhere. ${ }^{6}$ The recruitment of wildtype ataxin-3 into NIIs followed by ubiquitin may be a common mechanism in the formation of these nuclear inclusions. Interestingly, a recent study showed that wild-type huntingtin can attenuate the cellular toxicity of huntingtin in vivo with expanded polyglutamine. ${ }^{12}$ The recruitment of wild-type ataxin-3 into these nuclear inclusions might therefore be one of the cellular reactions aimed at counteracting noxious stimuli of extracellular origin. We do not know yet whether recruitment of ataxin-3 
into NIIs triggered by the abnormal expansion of the CAG repeat in the ataxin-3 gene is mediated by the same mechanism as in Marinesco bodies. The consistent presence of wild-type ataxin-3 surrounded by ubiquitin in Marinesco bodies and in NIIs of MJD/SCA3 suggests that a process involving both ataxin-3 and ubiquitin may be shared at least partly by these two different conditions.

In conclusion, the present study showed the consistent recruitment of ataxin-3 into Marinesco bodies surrounded by ubiquitin as previously seen in SCA3/MJD, ${ }^{6}$ suggesting that these inclusions are formed to reduce cellular stress. The absence of other polyglutamine proteins in Marinesco bodies suggests that the mechanism of recruitment does not involve polyglutamine alone but also a specific feature of the ataxin-3.

We thank Dr M Ruberg (INSERM U289, Hôpital de la Salpêtrière, France) for critical reading of the manuscript and Professor C Duyckaerts (Laboratoire de Neuropathologie Raymond Escourolle, Hôpital de la Salpêtrière, France) for statisti$\mathrm{cal}$ analyses. This work was supported partly by grants for Priority Area Research A, Ministry of Education, Science and Culture (HM) and Research on Human Genome, Tissue engineering and Food Biotechnology from the Ministry of Health and Welfare (HM, KI), from the Human Science Foundation (HM, TU), and from the Japan Society for the Promotion of Science (TU, HF, JT).

1 Zoghbi HY, Orr HT. Glutamine repeats and neurodegeneration. Anпu Rev Neurosci 2000;23:217-47.
2 Koyano S, Uchihara T, Fujigasaki $\mathrm{H}$, et al. Neuronal intranuclear inclusions in spinocerebellar ataxia type 2: triple-labeling immunofluorescent study. Neurosci Lett 1999;273:117-20.

3 Ishikawa K, Fujigasaki $\mathrm{H}$, Saegusa $\mathrm{H}$, et al. Abundant expression and cytoplasmic aggregations of $\alpha-1 \mathrm{~A}$ voltagedependent calcium channel protein associated with neurodegeneration in spinocerebellar ataxia type 6. Hum Mol Genet 1999;8:1185-93.

4 Perez MK, Paulson HL, Pendse SJ, et al. Recruitment and the role of nuclear localization in polyglutamine-mediated aggregation. F Cell Biol 1998;143:1457-70.

5 Uchihara T, Fujigasaki H, Koyano S, et al. Non-expanded polyglutamine proteins into intranuclear inclusions of hereditary ataxias: triple labelling immunofluorescent study. Acta Neuropathol (in press: DOI10.1007/s004010/ 00364).

6 Fujigasaki H, Uchihara T, Koyano S, et al. Ataxin-3 is translocated into the nucleus for the formation of intranuclear inclusions in normal and Machado-Joseph disease brains. Exp Neurol 2000;165:248-56.

7 Shiraki H, Yamamoto T. Histochemical aspects of hepatocerebral diseases. Shinkei Shinpo (Advances in Neurology) 1960;5:73-101.

8 Yuen, P, Baxter DW. The morphology of Marinesco bodies (paranucleolar colpuscles) in the melanin-pigmented nuclei of the brain-stem. 7 Neurol Neurosurg Psychiatry 1963; 26:178-83.

9 Hirai S. Ageing of the substantia nigra-histological and histochemical studies. Shinkei Shinpo (Advances in Neurology) 1965:12:845-9.

10 Bancher $\mathrm{CH}$, Lassman $\mathrm{H}$, Budka $\mathrm{H}$, et al. An antigenic profile of Lewy bodies: immunocytochemical indication for protein phosphorylation and ubiquitination. $\mathcal{F}$ Neuropathol protein phosphorylation and
Exp Neurol 1989;48:81-93.

11 Alves-Rodrigues A, Gregori L, Figueiredo-Pereira ME. Ubiquitin, cellular inclusions and their role in neurodegeneration. Trends Neurosci 1998;21:516-20.

12 Leavitt BR, Guttman JA, Hodgson JG, et al. Wild-type Huntingtin reduces the cellular toxicity of mutant Huntingtin in vivo. Am f Hum Genet 2001;68:313-24. 